\title{
Impact of Timing of Palatal Repair on Resonance, Understandability, and Acceptability in Children with Repaired Cleft Lip and Palate
}

\author{
M Pushpavathi *, Ajish K Abraham, SR Mahadeva Prasanna and Girish KS \\ All India Institute of Speech and Hearing, Mysuru, India
}

Submission: May11, 2018; Published: June 12, 2018

*Corresponding author: M Pushpavathi, Department of Speech-Language Pathology, All India Institute of Speech and Hearing (AIISH), Manasagangothri, Mysuru, 570006, India, Tel: 0821-2502570; Email: shivanna.pushpa@gmail.com

\section{Introduction}

Cleft lip and palate (CLP) is one of the most common congenital birth defects in India. The commonly noticeable speech errors in children with CLP are abnormalities in oral resonance, nasal air emission and presence of compensatory articulation errors which affects the overall intelligibility of speech. Children with CLP require early surgical intervention to establish appropriate oral motor skills that are adequate for normal speech production. This will re-establish a normal facial appearance and help in the acquisition of normal speech and language development. The speech and language development of the children with CLP depends on the age at which they undergo palatal surgery the type of cleft, the severity of the cleft, health condition of the child and socio-economic status. Researchers suggested that the ideal age for palatal repair is 3 to 6 months [8]. This recommendation is based on the theory that the palate must be functional when palate-related sounds are first learned to avoid poor speech development and integration. As the postoperative edema results in limited mobility of the repaired palate for an additional 3 to 6 months, it was advocated that the palatal repair should be done at 3 to 6 months of age so that the palate can function normally by 9 to 12 months [1-5].

Over decades, researchers have opined that an early cleft palate repair may reduce hypernasality, decrease the likelihood of the child developing compensatory articulation errors and help in better functioning of velopharyngeal port and improve speech intelligibility. A study was conducted describing nasal air emission, hypernasality, articulation and speech intelligibility in 38 children with early palate closure (before 18 months) and 30 children with delayed palatal closure (after 18 months) at the mean age of 4.3 years. The early closure group had less incidence of nasal air emission, hypernasality, articulation errors and significantly better speech intelligibility than the delayed closure group [5].

Similarly, another study evaluated speech acceptability, articulation, nasal resonance, and nasal air escape in 100 children with RCLP at the age of 5 years, who underwent a very early palate repair (mean age-16.4 weeks). The stimuli used were Edinburgh articulation test, a non-standardised test of articulation, conversational sample based on picture description, and counting from 1 to 10 . The results showed that 87 children had acceptable speech, 13 had unacceptable speech, 9 had poor articulation, 7 had moderate to severe nasal resonance and 9 had moderate to severe nasal escape. The study concluded that the early surgical intervention provides better speech outcome [3].

A comparative study evaluated articulation, phonation, nasal emission, nasal resonance, speech intelligibility and substitution patterns in 44 individuals (14 to 23 years) with RCLP across early palatal closure group and delayed palatal closure group, where 20 participants were there in early palatal closure group (at an average age of 10.6 months) and 18 participants were there in delayed palatal closure group (at an average age of 48.6 months). Edinburgh articulation test and taped van de mark sentences were administered to test articulation. Nasal emission, nasal resonance, and speech intelligibility were rated on a 4 point severity rating scale using simple words, sentences, and spontaneous speech. The results showed that the delayed palatal closure group performed significantly poorer in articulation, phonation, oral resonance and speech intelligibility than early palatal closure group The study concluded that delayed closure of the hard palate significantly reduces the chance for normal speech development [6-15].

Recently, an investigation compared resonance, airflow and articulation characteristics of 15 Ugandan participants with CLP who had delayed ( $\geq 8$ years) primary palatal closure, across age and gender-matched Ugandan participants without CLP with the mean age of 18.10 years. The participants were made to repeat the 12 oral and 3 nasal sentences (from SNAP test). Using the video recordings of the above stimuli, perceptual evaluation of hypernasality and nasal air emission were done on a 4-point rating scale. Standardized picture-naming Photo Articulation 
Test was used to assess articulation. Nasalance values were also calculated using Nasometry. It was found that individuals with CLP had a significantly higher prevalence of hypernasality, articulation errors and higher nasalance values for all oral and oro-nasal speech samples. The study revealed that delayed palatal repair is insufficient to eliminate nasal airflow errors, resonance abnormalities, and articulation disorders which leads to unintelligible speech [2].

In an Indian context, the speech outcome of 131 individuals with CLP who underwent primary palate repair after the age of 10 years were evaluated. Baseline assessment was done and all the individuals were counseled, oriented, and demonstrated the correct place and manner of articulation for the phonemes misarticulated by them. Though postoperative speech samples assessment revealed improvement in speech intelligibility, there were residual speech problems in most of the individuals with repaired cleft lip and palate (RCLP), requiring further evaluation and appropriate treatment. The study concluded that more residual speech problems are found with late palatal surgical intervention [12].

Another Indian study compared the speech characteristics of three toddlers with RCLP who underwent surgery before 1.6 years of age (Early Intervention Group) with three toddlers with RCLP who underwent surgery after 1.6 years of age (Delayed Intervention Group). All the toddlers had Kannada as their native language and they were in the age range of 2-3 year. The baseline was established and measures such as type and pattern of the vowels and consonant inventory were analyzed for the toddlers in both the groups. The post-therapy measurement was done after 20 Speech and language therapy sessions. The results revealed that the early intervention group had better and diversified phonetic inventory when compared to the delayed intervention group. It was concluded that the timing of the surgery of cleft lip and palate plays an important role in shaping the future communicative abilities [13].

There are few contraindications for this viewpoint on early palatal surgery, where investigations have reported no significant difference in resonance and speech intelligibility between early and late palatal repair. A study compared 12 Ugandan children with CLP following early palatal repair (before 6 months) and 12 Belgian individuals with later palatal repair (after 6 months) where no significant difference as found in resonance and speech intelligibility between early and late palatal repair group. The study revealed that resonance and speech intelligibility of children following palatal repair before and after 6 months of age seem to be at least comparable [11].

Also, researchers have argued that the palate should be repaired later in the child's life or after the speech has developed, because a palatoplasty may hinder the growth and development of maxilla [9]. The operative procedures to repair the primary palate may be harmful to maxillary growth causing alveolar collapse and subsequent overall facial growth impairment [1].
After investigating the maxillary growth of 125 individuals with cleft palate, a study reported that hard palate repair in the first 2 years of life causes greater dental arch collapse than the hard palate repair at 3 or 4 years [14]. But there are also studies which do not support the finding that early cleft palate surgery hinders the maxillary growth.

However, even with early surgical repair of the cleft, speech errors persist in most of the children with CLP. These speech characteristics related to articulation and resonance problems in children with CLP requires a detailed assessment by SpeechLanguage Pathologists (SLPs). Detailed speech and language assessment include both perceptual as well as instrumental evaluations like Nasometry, Nasal Visualization System evaluation, Naso-endoscopy etc, but the perceptual assessment is considered as the gold standard for the analysis of speech [10]. Articulation, resonance and speech intelligibility are crucial measures of disordered speech and an important goal of therapeutic intervention for children with RCLP. Thus carrying out the perceptual judgment of speech parameters is important in order to measure the speech outcomes and to determine appropriate treatment plans.

There have been limited attempts in the Indian context to study the impact of timing of surgery on resonance, speech understandability and speech acceptability in children with RCLP across stimuli. Hence, the present study aims to explore these parameters in the early surgical group and delayed surgical group across different stimuli. The objectives of the present study are as follows:

i. To compare resonance, speech understandability and speech acceptability across early intervention group and delayed intervention group in words

ii. To investigate resonance, speech understandability and speech acceptability across early intervention group and delayed intervention group in sentences

iii. To compare resonance, speech understandability, and speech acceptability across stimuli within early intervention group and delayed intervention group.

\section{Method}

\section{Participants}

In the present study, 16 Kannada speaking children with non syndromic RCLP in the age range of 6 to 12 years were considered. 8 children were in the early intervention group (EIG - who underwent surgery before 1.6 years of age) and 8 children were in the delayed intervention group (DIG - who underwent surgery after 1.6 years of age) with an equal number of males and females, as shown in Table 1. The language abilities of all the participants with repaired cleft palate were age adequate. Individuals with other associated problems like hearing loss, intellectual disability, nasal pathologies, and syndromes were excluded from this study. 
Table 1: Participants details in EIG and DIG.

\begin{tabular}{|c|c|c|c|}
\hline & EIG & DIG & Total \\
\hline Males & 4 & 4 & 8 \\
\hline Females & 4 & 4 & 8 \\
\hline Total & 8 & 8 & 16 \\
\hline
\end{tabular}

\section{Materials and procedure}

The speech stimuli used for the present study consisted of 10 meaningful words and 10 meaningful oral sentences loaded with pressure consonants in Kannada Language [7]. Participants were made to repeat the stimuli and the responses were recorded using Brüel and Kjær Sound Level Meter (Type 2250s Hand Held Analyzer) in a sound-treated room. The recorded speech samples were presented to 3 experienced Speech Language Pathologists (SLPs) who were working in the research and clinical area related to cleft lip and palate, for perceptual evaluation. A standardized 4 point rating scale was used for perceptual judgment of resonance, understandability, and acceptability [6], where $0=$ normal, $1=$ mild, $2=$ moderate and $3=$ severe. Each individual's total score varied from 0 to 30 in both words and in sentences. Both oral and written instructions for perceptual evaluation were provided to the SLPs. SLPs were blindfolded to the objectives of the study, participants of the study, and the stimuli were presented through the headphones for rating in a quiet room situation. The maximum occurrence of a rating among 3 judges was considered for further analysis.

\section{Statistical Analysis}

Obtained data were subjected to statistical computation using IBM Statistical Package Social Sciences software version 20. Average mean and standard deviation of resonance, understandability, and acceptability in both words and sentences were calculated separately. Shapiro-Wilk test of normality was applied to check the normality, where the test revealed the normal distribution of the data $(\mathrm{p}>0.05)$. Independent samples t-test was carried out to check if there was any difference in resonance, understandability, and acceptability in both words and sentences across EIG and DIG. Cronbach's alpha was calculated for words and sentences which showed higher interjudge reliability among three judges $(\alpha>0.70)$.

\section{Results}

The present study is one of the preliminary attempts in the Indian context to investigate the impact of timing of surgery on resonance, speech understandability and speech acceptability in RCLP across stimuli. The obtained results are discussed under following sub-sections.

Comparison of resonance, speech understandability and speech acceptability across early intervention group and delayed intervention group in words

The total mean score and standard deviation of resonance, understandability, and acceptability were calculated in words for EIG and DIG, as shown in Table 2.
Table 2: Mean and standard deviation of raw scores for words across EIG and DIG ( $\left.{ }^{*} \mathrm{p} \leq 0.05\right)$.

\begin{tabular}{|c|c|c|c|c|c|}
\hline & \multicolumn{2}{|c|}{ EIG } & \multicolumn{2}{c|}{ DIG } & \\
\hline & Mean & SD & Mean & SD & p-value \\
\hline Resonance & 11.38 & 2.66 & 16.88 & 4.39 & ${ }^{*} 0.009$ \\
\hline Understandability & 12.75 & 5.94 & 15.38 & 4.50 & 0.336 \\
\hline Acceptability & 14.25 & 4.68 & 17.88 & 3.60 & 0.105 \\
\hline
\end{tabular}

(EIG- Early Intervention Group; DIG- Late Intervention Group)

The total mean scores of resonance, understandability, and acceptability rated by the 3 SLPs were comparatively greater in DIG than in EIG. The percentage of mean scores of resonance, understandability, and acceptability in words were calculated in DIG and EIG and it is represented in Figure 1. The results clearly indicated that, as the values of all the 3 parameters increased, the performance of the participants deteriorated, and conversely, lower scores indicated a better performance. Here, EIG performed better than DIG in resonance, speech understandability, and speech acceptability. Independent two-sample t-test was conducted to compare resonance, understandability, and acceptability across EIG and DIG in words. Among all the three variables, it was observed that there was a significant difference only in resonance $[t(14)=3.028, p<0.05]$. There was no significant difference found in understandability $[\mathrm{t}(14)=0.995, \mathrm{p}>0.05]$ and acceptability $[\mathrm{t}(14)=1.735, \mathrm{p}>0.05]$ across EIG and DIG.

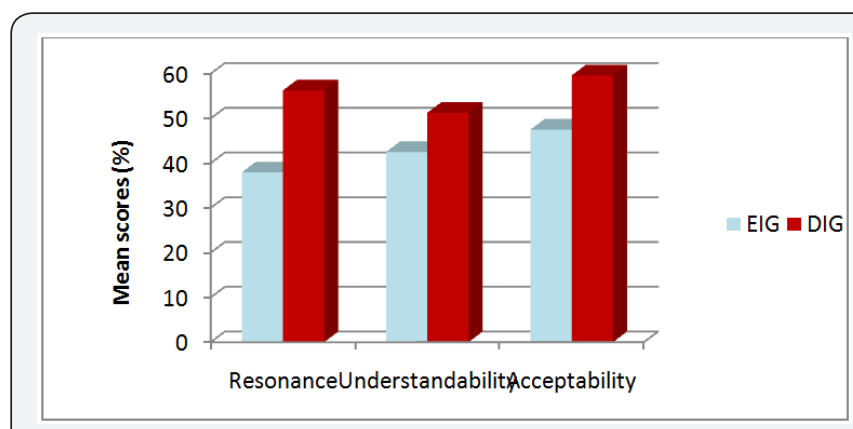

Figure 1: The percentage of mean scores in EIG and DIG for words.

Comparison of resonance, speech understandability and speech acceptability across early intervention group and delayed intervention group in sentences

The total mean score and standard deviation of resonance, understandability, and acceptability were calculated for sentences in EIG and DIG, as shown in Table 3. The mean and standard deviation scores in sentences showed a similar trend like that of words. The total mean scores of resonance, understandability, and acceptability rated by the 3 SLPs were comparatively greater in DIG than in EIG. The percentage of mean scores of resonance, understandability, and acceptability in sentences were calculated in DIG and EIG, as represented in Figure 2. EIG performed better than DIG in resonance, speech 


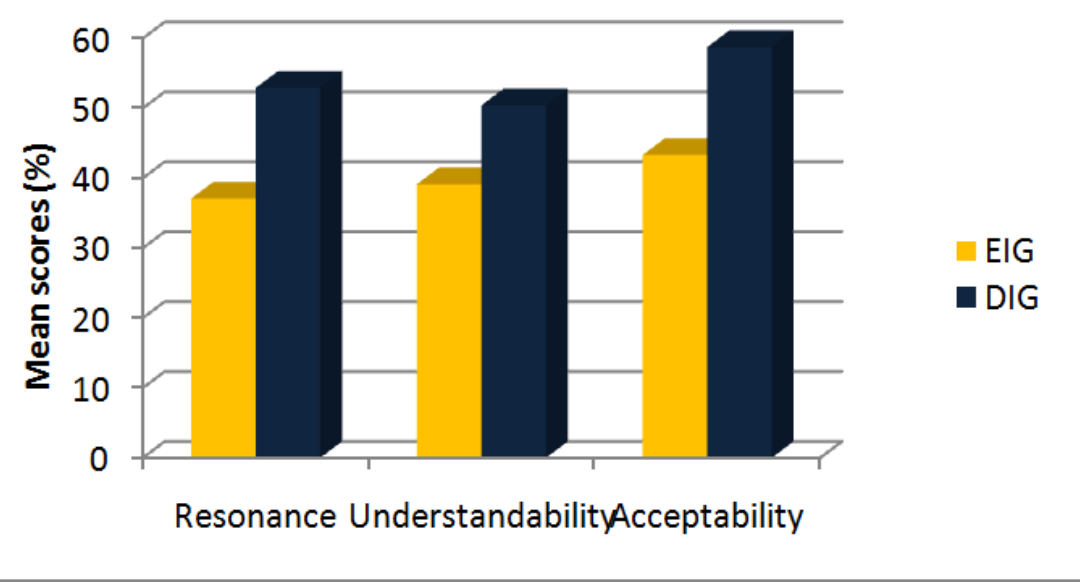

Figure 2: The percentage mean scores in EIG and DIG for sentences.

Table 3: Mean and standard deviation of raw scores in words across EIG and DIG ( $\left.{ }^{*} p \leq 0.05\right)$.

\begin{tabular}{|c|c|c|c|c|c|}
\hline & \multicolumn{2}{|c|}{ EIG } & \multicolumn{2}{|c|}{ DIG } & SD \\
\hline & Mean & SD & Mean & 3.52 & $* 0.007$ \\
\hline Resonance & 11.13 & 2.35 & 15.88 & 4.08 & 0.189 \\
\hline Understandability & 11.75 & 5.57 & 15.13 & 4.71 & 0.106 \\
\hline Acceptability & 13 & 5.92 & 17.63 & & \\
\hline
\end{tabular}

(EIG- Early Intervention Group; DIG- Late Intervention Group)

Independent two-sample t-test was conducted to compare resonance, understandability, and acceptability across EIG and DIG in sentences. It was observed that among all the three variables there was a significant difference only in resonance $[\mathrm{t}(14)=3.170, \mathrm{p}<0.05]$. There was no significant difference found in understandability $[\mathrm{t}(14)=0.995, \mathrm{p}>0.05]$ and acceptability [t(14) $=1.726, \mathrm{p}>0.05]$ across EIG and DIG.

To compare resonance, speech understandability and speech acceptability across stimuli within early intervention group and delayed intervention group

The total mean score and standard deviation of resonance, understandability, and acceptability were calculated for words and sentences within EIG and DIG as shown in Table 4. In both EIG and DIG, the total mean scores of resonance, understandability, and acceptability rated by the 3 SLPs were comparatively greater in words than in sentences. Paired t-test was administered to compare resonance, understandability, and acceptability across words and sentences within EIG and DIG. In EIG, it was observed that there was no significant difference found across words and sentences among all the variables i.e., resonance $[\mathrm{t}(7)=0.775, \mathrm{p}>0.05]$ understandability $[\mathrm{t}(7)=0.499, \mathrm{p}>0.05]$ and acceptability $[\mathrm{t}(7)=0.180, \mathrm{p}>0.05]$. In DIG also there was no significant difference across words and sentences among all the variables i.e., resonance [ $\mathrm{t}(7)=0.571, \mathrm{p}>0.05]$ understandability $[\mathrm{t}(7)=0.228, \mathrm{p}>0.05]$ and acceptability $[\mathrm{t}(7)=0.261, \mathrm{p}>0.05]$.

Table 4: Mean and standard deviation of raw scores across words and sentences in EIG and DIG (* $\mathrm{p} \leq 0.05)$.

\begin{tabular}{|c|c|c|c|c|c|c|c|}
\hline & & \multicolumn{2}{|c|}{ EIG } & \multirow{2}{*}{ p value } & \multicolumn{2}{|c|}{ DIG } & \multirow{2}{*}{ p value } \\
\hline & & Mean & SD & & Mean & SD & \\
\hline \multirow{2}{*}{ Resonance } & Words & 11.38 & 2.66 & \multirow{2}{*}{0.775} & 16.88 & 4.39 & \multirow{2}{*}{0.586} \\
\hline & Sentences & 11.13 & 2.35 & & 15.88 & 3.52 & \\
\hline \multirow{2}{*}{ Understandability } & Words & 12.75 & 5.94 & \multirow{2}{*}{0.499} & 15.38 & 4.50 & \multirow{2}{*}{0.826} \\
\hline & Sentences & 11.75 & 5.57 & & 15.13 & 4.08 & \\
\hline \multirow{2}{*}{ Acceptability } & Words & 14.25 & 4.68 & \multirow{2}{*}{0.180} & 17.88 & 3.60 & \multirow{2}{*}{0.802} \\
\hline & Sentences & 13 & 5.92 & & 17.63 & 4.71 & \\
\hline
\end{tabular}

(EIG- Early Intervention Group; DIG- Late Intervention Group) 


\section{Discussion}

The presentstudy investigated the impact of timing of surgery on resonance, speech understandability and speech acceptability in RCLP across EIG and DIG. In both words and sentences, DIG had higher mean scores in resonance, understandability, and acceptability than EIG but it was statistically significant only in resonance. This shows that hypernasality is significantly more in DIG than in EIG. This result is in consonance with the previous studies which also found a significantly higher prevalence of hypernasality in Ugandan participants with CLP who had delayed primary palatal closure age [2]. The result of the present study also supports the study who reported significantly increased hypernasality in delayed palatal closure group [15]. The present study supports the findings of the previous investigation which reported that the early complete closure group had a significantly lesser incidence of hypernasality, nasal air emission than the delayed closure group [5]. This suggests that early surgical intervention has a direct impact on reducing hypernasality in children with CLP with better speech outcome.

Among both the stimuli, speech understandability and speech acceptability were reduced in the DIG when compared to the EIG but it was not statistically significant. This result supports the findings of the earlier study which reported no significant difference in speech intelligibility across early palatal repair group and late palatal group [11]. But the result of the present study is in contraindication with the findings of few previously conducted studies which reported that speech intelligibility of individuals with delayed palatal repair was significantly poorer than early palatal repair $[5,15]$.

Though resonance and speech intelligibility are inter-related and have a direct impact on each other, significant difference was found only in resonance but not in speech understand ability and speech acceptability, across EIG and DIG. This resultant difference in hypernasality alone could be because the overall speech intelligibility in children with CLP is affected by many factors such as articulatory abilities, the rate of speech and resonance among other factors. So there is a need for the inclusion of these parameters in the assessment protocol.

Among the factors that can influence the perceptual judgment of hypernasality, understandability and acceptability, type of speech stimuli is considered as a major factor. The present study used words and sentences for the perceptual evaluation and effect of stimuli was also investigated. In both EIG and DIG, the total mean scores of resonance, understandability, and acceptability were comparatively greater in words than in sentences but there was no significant difference found across stimuli among all the variables. This result is in consensus with the findings of an Indian study where the authors reported no significant difference in the speech intelligibility scores across words and sentences [4]. But they also opined that type of stimuli influences the speech intelligibility. There are many other important factors which contribute to the above results. The present study considered only 16 participants, a significant difference in speech understandability, speech acceptability and stimuli effect could have been obtained with the inclusion of larger sample size and with the inclusion of spontaneous speech as one of the test stimuli.

\section{Conclusion}

The present study concludes that early surgical intervention has a direct impact on reducing hypernasality in children with CLP. It also found that the parameter resonance alone is not responsible for reduced overall speech understandability and speech acceptability. The study also provides implication that working on improving resonance alone in speech therapeutic intervention does not help in the improving speech intelligibility.

\section{Acknowledgment}

This is a part of ongoing research on "NASOSPEECH: Development of Diagnostic System for Severity Assessment of the Disordered Speech", funded by the Department of Biotechnology (DBT - No. SH/PL/DBT (AKA)/2016-17), Government of India. The authors would like to thank DBT for funding the project. The authors would also like to thank Dr. S.R.Savithri, Director, All India Institute of Speech and Hearing, Mysuru, for providing the infrastructure to carry out the study.

\section{References}

1. Bardach J, Roberts DM, Russell Yale MD, Rosewall D, Mooney M (1980) The influence of simultaneous cleft lip and palate repair on facial growth in rabbits. Cleft Palate J 17(4): 309-318.

2. Bruneel L, Luyten A, Bettens K, D'haeseleer E, Dhondt C, et al. (2017) Delayed primary palatal closure in resource-poor countries: Speech results in Ugandan older children and young adults with cleft (lip and) palate. J Commun Disord 69: 1-14.

3. Copeland M (1990) The effects of very early palatal repair on speech. Br J Plast Surg 43(6): 676-682.

4. Gnanavel K, Pushpavathi M (2012) Effect of type of cleft and speech stimuli on speech intelligibility in individuals with cleft lip and palate. J All India Inst Speech Hear 31: 33-39.

5. Henningssson G, Karling J (1984) Early or Late Surgery of the Hard Palate? A Comparison of Speech at Four Years of Age. Plast Reconstr Surg.

6. Henningsson G, Kuehn DP, Sell D, Sweeney T, Trost-Cardamone JE, et al. (2008) Universal parameters for reporting speech outcomes in individuals with cleft palate. Cleft Palate Craniofac J 45(1): 1-17.

7. Jayakumar T, Pushpavathi M (2005) Normative score for nasometer in Kannada. Student research at AIISH (Articles based on dissertation done at AIISH) 7: 44-53.

8. Kaplan EN (1975) Soft palate repair by levator muscle reconstruction and a buccal mucosal flap. Plast Reconstr Surg 56(2): 129-136.

9. Kremenak CR, Huffman WC, Olin WH (1970) Maxillary growth inhibition by mucoperiosteal denudation of shelf bone in non-cleft beagles. Cleft Palate Craniofac J 7: 817.

10. Kuehn DP, Moller KT (2000) Speech and language issues in the cleft palate population: The state of the art. Cleft Palate Craniofac J 37(4): 348-348. 
11. Luyten A, Bettens K, D’haeseleer E, De Ley S, Hodges A, et al. (2014) The impact of palatal repair before and after 6 months of age on speech characteristics. Int J Pediatr Otorhinolaryngol 78(5): 787-798.

12. Murthy J, Sendhilnathan S, Hussain SA (2010) Speech outcome following late primary palate repair. Cleft Palate Craniofac J 47(2): 156-161.

13. Pushpavathi M, Kavya V, Akshatha V (2017) Impact of timing of surgery on the development of speech in toddlers with repaired cleft lip and palate. Proceedings of National Symposium on Acoustics 46: 41-51.
14. Rayner HH (1925) The operative treatment of cleft-palate.: A record of results in 125 consecutive cases. Lancet 205(5303): 816-818.

15. Rohrich RJ, Rowsell AR, Johns DF, Drury MA, Grieg G, et al. (1996) Timing of hard palatal closure: a critical long-term analysis. Plast Reconstr Surg 98(2): 236-246.

\section{Your next submission with Juniper Publishers will reach you the below assets}

- Quality Editorial service

- Swift Peer Review

- Reprints availability

- E-prints Service

- Manuscript Podcast for convenient understanding

- Global attainment for your research

- Manuscript accessibility in different formats

( Pdf, E-pub, Full Text, Audio)

- Unceasing customer service

Track the below URL for one-step submission https://juniperpublishers.com/online-submission.php 